\title{
Role of Surface Friction on Shallow Nonprecipitating Convection
}

\author{
SEUNG-BU PARK \\ Department of Earth and Environmental Engineering, Columbia University, New York, New York, and Institute for \\ Basic Science Center for Climate Physics, Pusan National University, Busan, South Korea \\ STEVEN BÖING \\ School of Earth and Environment, University of Leeds, Leeds, United Kingdom \\ PIERRE GENTINE \\ Department of Earth and Environmental Engineering, and Earth Institute, Columbia University, New York, New York
}

(Manuscript received 5 April 2017, in final form 22 October 2017)

\begin{abstract}
The role of surface friction on shallow nonprecipitating convection is investigated using a series of large-eddy simulations with varying surface friction velocity and with a cloud identification algorithm. As surface friction intensifies, convective rolls dominate over convective cells and secondary overturning circulation becomes stronger in the subcloud layer, thus transporting more moisture upward and more heat downward between the subcloud and cloud layers. Identifying individual clouds, using the identification algorithm based on a threedimensional topological analysis, reveals that intensified surface friction increases the number of clouds and the degree of tilting in the downstream direction. Highly intensified surface friction increases wind shear across the cloud base and induces cloud tilting, which leads to a vertically parabolic profile of liquid water mixing ratio instead of the classical two-layer structure (conditionally unstable and trade inversion layers). Furthermore, cloud tilting induces more cloud cover and more cloud mass flux much above the cloud base (e.g., $0.8<z<1.2 \mathrm{~km}$ ), but less cloud cover and less cloud mass flux in the upper cloud layer (e.g., $z>1.2 \mathrm{~km}$ ) because of increased lateral entrainment rate. Similarly, profiles of directly measured entrainment and detrainment rates show that detrainment in the lower cloud layer becomes smaller with stronger surface friction.
\end{abstract}

\section{Introduction}

Boundary layer clouds are still one of the largest uncertainties in weather and climate prediction despite their importance in the radiative feedback and energy/ hydrological cycle (Bony et al. 2006; Nuijens et al. 2015). The uncertainty comes mainly from insufficient understanding of how turbulence and microphysics generate clouds in diverse environments (e.g., various wind shear, thermal stratification, and humidity) and how clouds mix with the environment (de Rooy et al. 2013).

In the subcloud layer below the cloud base, a variety of turbulent organized structures, so-called coherent structures, appear and transport momentum, heat, and moisture upward and downward. For example, sweeps and ejections stir surface-layer air, and thermal updrafts pump up the mixed heat and moisture (Raupach 1981;

Corresponding author: Seung-Bu Park, sseungbu@gmail.com
Katul et al. 1997; Couvreux et al. 2010). Convective cells (rolls), composed of scattered (aligned) thermal updrafts and environmental subsidence, span the whole subcloud layer and thus bring the surface-layer heat and moisture upward up to the inversion layer, further generating clouds (Park et al. 2016). The trapped/ overshooting updrafts and environmental subsidence induce compensating overturning circulations, secondary to the geostrophic wind, and the secondary circulations transport pumped-up/entrained air all through the boundary layer (Stull 1988). Thus, the convective cells and rolls play a major role in transporting bottomemitted scalars and entraining free-tropospheric air (Sullivan et al. 1998; Gentine et al. 2015).

The temporal and spatial distributions of the above two coherent structures are controlled by two key factors: surface friction and buoyancy (Sykes and Henn 1989; Moeng and Sullivan 1994; Salesky et al. 2016). The temporal transition from the convective rolls to cells is 
frequently observed in the morning-to-afternoon continental dry convection when the diurnally increasing sensible heat flux dominates surface friction (Weckwerth et al. 1999). The spatial transition between the convective cells and rolls can be found in regions where surface roughness abruptly changes. For example, convective cells become rolls and generate cloud trails behind small islands (Matthews et al. 2007), and convective rolls are dominant over the urban surfaces (Kropfli and Kohn 1978; Park and Baik 2014). However, most of such surface roughness change is often accompanied by changes in surface albedo and Bowen ratio, therefore limiting the understanding and observation of the sole impact of surface friction.

Surface friction controls the pattern of subcloud-layer circulation and moisture transport therein and the number and morphology of clouds. Although the impact of surface friction change within the subcloud layer can be parameterized by the roughness length, drag (Grant and Brown 1999), and entrainment heat flux (Troen and Mahrt 1986; Moeng and Sullivan 1994) in numerical weather and climate models (in which individual surface eddies and thermal updrafts are not explicitly resolved), its impact on clouds is poorly understood and thus cannot be accurately represented yet. The entrainment and detrainment rates in particular, which quantify the mixing of clouds and the environment and determine the vertical distribution of mass flux (de Rooy and Siebesma 2008), need to be refined to accurately reflect the impact of shear.

In this study, the role of surface friction on nonprecipitating shallow convection is investigated by performing a series of large-eddy simulations with varying surface friction velocity in isolation from other changes such as Bowen ratio changes. The paper is organized as follows. Section 2 describes the simulation setup and cloud identification method. The impact of surface friction on the subcloud and cloud layers is presented in section 3 . A summary and conclusions are given in section 4.

\section{Methodology}

\section{a. Large-eddy simulation}

In this study, we use the University of California, Los Angeles, large-eddy simulation (UCLA-LES) model (Stevens et al. 1999, 2005; Stevens and Seifert 2008) to simulate nonprecipitating shallow cumulus convection. The LES model solves implicitly filtered prognostic equations of velocity components $(u, v, w)$, liquid water potential temperature $\theta_{l}$, and total water mixing ratio $r_{t}$. The equations of prognostic variables on a staggered three-dimensional grid are integrated using a third-order
Runge-Kutta scheme (Wicker and Skamarock 2002), and the subgrid-scale fluxes of momentum and the thermodynamic variables are parameterized using the Smagorinsky model. The reversible conversion between water vapor and liquid water (cloud) is modeled in this nonprecipitating cumulus simulation, and the liquid water mixing ratio is diagnostically calculated using a saturation adjustment scheme. The initial sounding, external forcing (subsidence, large-scale drying, and geostrophic wind), and surface heat and moisture fluxes described in Siebesma et al. (2003) and Siebesma and Cuijpers (1995) are used to simulate the trade wind cumulus convection in the Barbados Oceanographic and Meteorological Experiment (BOMEX; Holland and Rasmusson 1973). The $512 \times 512 \times 144$ staggered grid points, spaced by $25 \mathrm{~m}$ in the $x$ (east-west) and $y$ (south-north) directions and 20$52.3 \mathrm{~m}$ in the $z$ (vertical) direction, cover $12.8 \times 12.8 \times$ $3.06 \mathrm{~km}^{3}$. A variable time step is used for the peak CFL number $\Delta t \max (|u / \Delta x|,|v / \Delta y|,|w / \Delta z|)$ not exceeding 0.5 , and the scaled time step is $\sim 1 \mathrm{~s}$ after spinup.

The surface friction velocity $u_{*}$ in the original intercomparison study (Siebesma et al. 2003) was fixed to $0.28 \mathrm{~m} \mathrm{~s}^{-1}$, and the surface momentum fluxes are calculated as $\left(\widetilde{u^{\prime \prime} w^{\prime \prime}}{ }_{0}, \bar{v}^{\prime \prime} w^{\prime \prime}{ }_{0}\right)=-u_{*}^{2} \times$ $\left(u_{\Delta z / 2} / \sqrt{u_{\Delta z / 2}^{2}+v_{\Delta z / 2}^{2}}, v_{\Delta z / 2} / \sqrt{u_{\Delta z / 2}^{2}+v_{\Delta z / 2}^{2}}\right)$, where the tilde and double prime indicate gridbox averaging (filtering) and subgrid-scale perturbation, respectively, and the subscript $\Delta z / 2$ indicates the center level of the lowest grids above the ground. In this study, to assess the impact of changes in surface friction and shear, we vary $u_{*}$ between 0.07 and $0.56 \mathrm{~m} \mathrm{~s}^{-1}$, while keeping the geostrophic wind the same: $\left(u_{g}, v_{g}\right)=(-10+1.8 \times$ $\left.10^{-3} z \mathrm{~m} \mathrm{~s}^{-1}, 0 \mathrm{~m} \mathrm{~s}^{-1}\right)$ where $z$ is height above ground level, and the same Coriolis parameter at $15^{\circ} \mathrm{N}$. The cases are named smooth S3, S2, and S1; control (CTL); and rough R1, R2, and R3 in order of $u_{*}$ magnitude (Table 1 ). The numbers in the names indicate the surface smoothness and roughness compared to the control case. The control and six $u_{*}$-altered cases are simulated for $6 \mathrm{~h}$, and the data for the last $2 \mathrm{~h}$ are sampled every $10 \mathrm{~min}$. In all cases, the kinematic sensible and latent surface heat fluxes are prescribed as constant and set to 9.46 and 153.01 $\mathrm{W} \mathrm{m}^{-2}$ as in Siebesma et al. (2003) to eliminate the impact of surface friction on surface heat and moisture fluxes. Indeed, otherwise, the subcloud layer would be quite different in terms of their mean quantities, and we could not understand the pure dynamical effect of surface friction change. Although these idealized simulations with fixed surface fluxes illustrate the sole dynamical impact of surface friction change well, in addition, we also performed simulations with surface fluxes calculated using Monin-Obukhov similarity theory, and the results are 
TABLE 1. The surface friction velocity, subcloud-layer height (where the minimum of virtual potential temperature flux occurs), ratio of subcloud-layer height and Obukhov length, ratio of surface friction velocity and Deardorff convective velocity scale, and the total number of identified clouds for 12 time instants in each case.

\begin{tabular}{|c|c|c|c|c|c|c|c|}
\hline & \multicolumn{7}{|c|}{ Case } \\
\hline & S3 & $\mathrm{S} 2$ & S1 & CTL & $\mathrm{R} 1$ & $\mathrm{R} 2$ & R3 \\
\hline$u_{*}\left(\mathrm{~m} \mathrm{~s}^{-1}\right)$ & 0.07 & 0.14 & 0.21 & 0.28 & 0.35 & 0.42 & 0.56 \\
\hline$z_{i}(\mathrm{~m})$ & 590 & 590 & 590 & 590 & 590 & 610 & 630 \\
\hline$z_{i} / L$ & 392.1 & 49.0 & 14.5 & 6.1 & 3.1 & 1.9 & 0.8 \\
\hline$u_{*} / w_{*}$ & 0.10 & 0.20 & 0.30 & 0.40 & 0.50 & 0.60 & 0.79 \\
\hline$N_{\text {cloud }}$ & 2248 & 2229 & 2283 & 2302 & 2250 & 2703 & 2776 \\
\hline
\end{tabular}

presented in appendix B. Altering the surface fluxes obviously strongly modifies the state of the subcloud and cloud layers as it strongly modifies the heat and moisture inputs of the simulations (appendix B, Fig. B1). We thus believe that those simulations cannot directly be used to understand and isolate the dynamical effect of momentum; hence, our strategy is to only modify the shear effect in the momentum budget. We also performed simulations without the Coriolis force (appendix B). The main change is that the heat and moisture can be transported up to higher levels (Fig. B1), but the overall shape of the vertical heat, moisture, and momentum transports is similar. Given that we are mostly interested in trade wind shallow convection and that the BOMEX case has been substantially evaluated, we thus decide to keep the same Coriolis parameter as those reference simulations.

\section{b. Identification of clouds}

To analyze the statistics of individual clouds (e.g., their size and number), we use an offline three-dimensional cloud identification algorithm. Similar to a number of previous studies (e.g., Dixon and Wiener 1993; Heus et al. 2009), this algorithm uses connected cloudy grid cells ( $r_{l} \geq 0.01 \mathrm{~g} \mathrm{~kg}^{-1}$, where $r_{l}$ is liquid water mixing ratio) to identify a cloud. However, clouds that consist of multiple fragments (e.g., the westernmost cloud in Fig. 5b) present a challenge for such cloud identification algorithms (Heus et al. 2009). One approach that is often taken is to further distinguish core regions (e.g., using high liquid water mixing ratio) and their associated regions. This can be done by introducing an additional criterion for cores (e.g., Dawe and Austin 2012; Heus and Seifert 2013). An alternative approach (Johnson et al. 1998; Yu and Yang 2017) is to use multiple thresholds. The current algorithm uses a third different approach based on a three-dimensional topological analysis of the field. This algorithm does not divide the clouds into cores and associated regions. Similar but more generic techniques exist in the literature on computational geometry (Carr et al. 2003), and topological methods have also been used to analyze volcanic plumes (Kuhn and Trömel 2015).

The algorithm starts by defining a binary mask $M$, which in our case indicates whether a cell contains liquid water $\left(r_{l} \geq 0.01 \mathrm{~g} \mathrm{~kg}^{-1}\right)$, and an associated value field $\psi$, which in our case is the field of liquid water mixing ratio. The mask determines for which grid cells a cloud number is identified, whereas the value field is used to divide

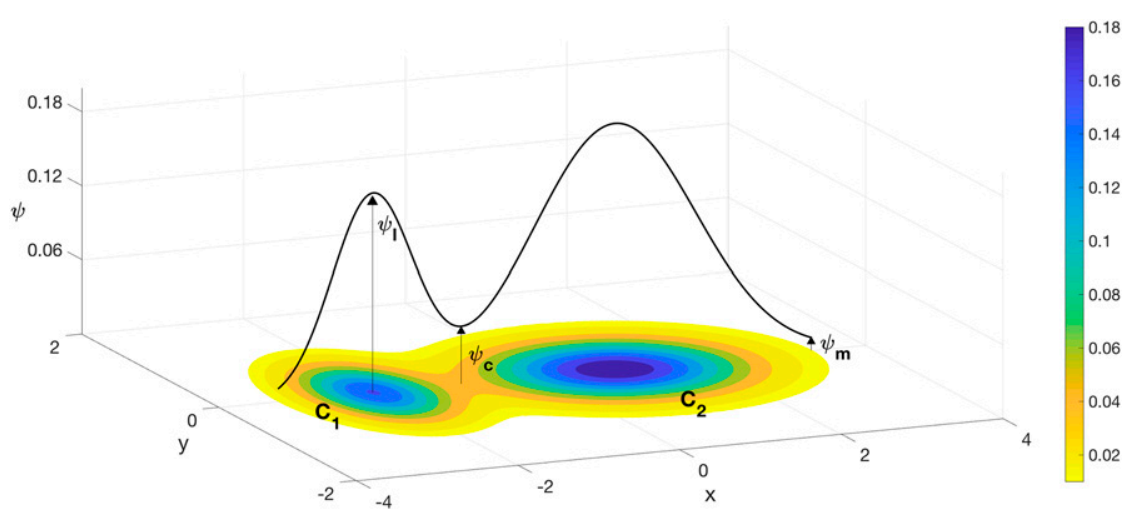

FIG. 1. A sketch explaining the merging algorithm using the different heights $\psi_{c}, \psi_{l}$, and $\psi_{m}$. The color shading illustrates the two-dimensional distribution of cloud fragments $C_{1}$ and $C_{2}$, whereas our algorithm uses three-dimensional data. 

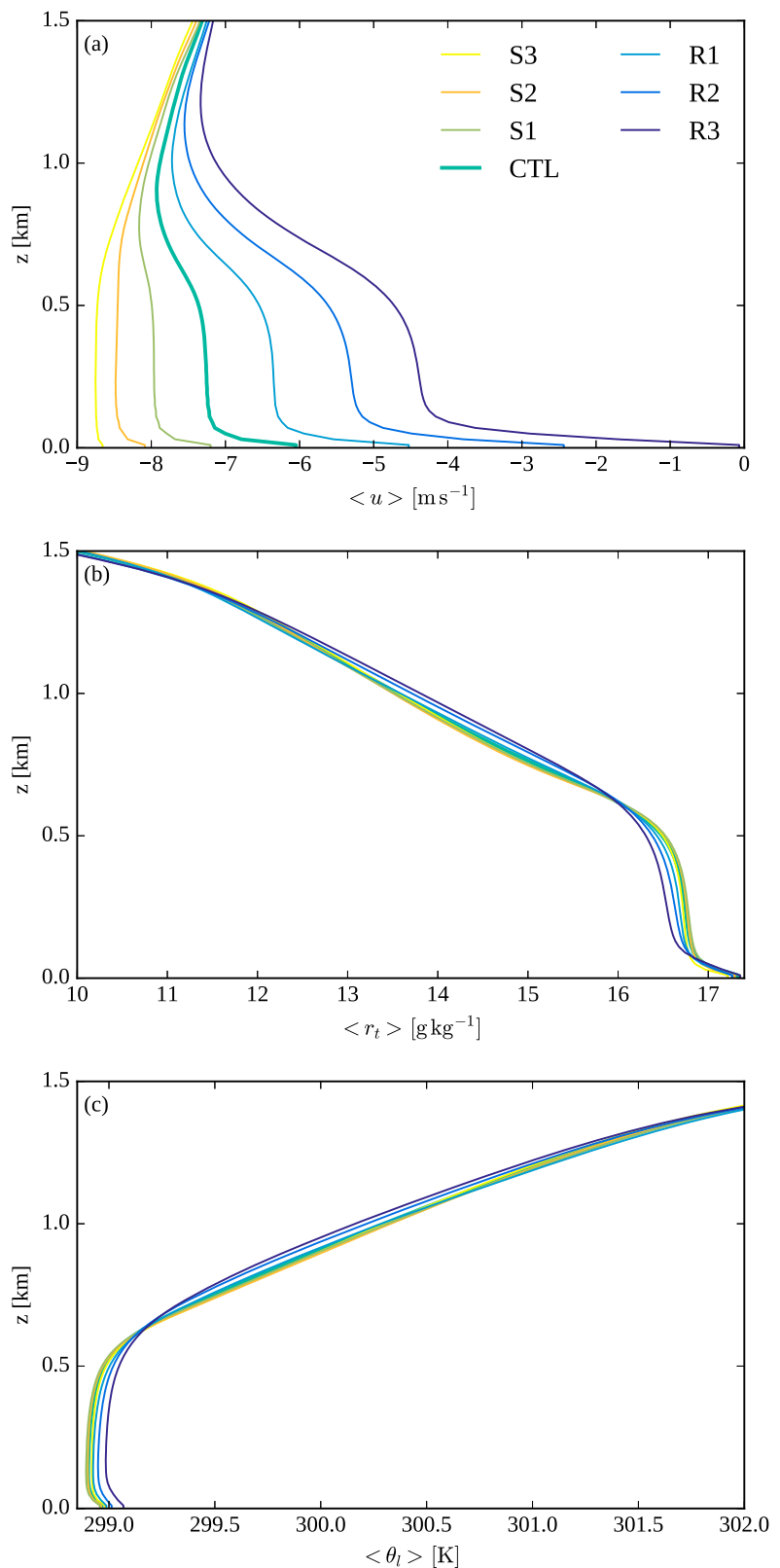

FIG. 2. Profiles of horizontally and temporally (last $2 \mathrm{~h}$ ) averaged (a) zonal velocity, (b) total water mixing ratio, and (c) liquid water potential temperature.

the field into different cloud fragments, which can border each other. This segmentation step is a two-step process: First, we associate each grid cell with a local maximum of $\psi$ and identify fragments that are topologically connected. The fragments can be thought of as watersheds (related to a local maximum instead of a local minimum). Subsequently, we merge the identified fragments in case they are associated with a relatively insignificant local maximum of $\psi$, as outlined below.
In the first fragment-identification step, we consider neighboring values $\psi(i \pm 1, j, k), \psi(i, j \pm 1, k)$, and $\psi(i, j, k \pm 1)$ for each cell. We determine the neighbor that corresponds to the steepest upward gradient (i.e., the neighbor with the highest value of $\psi$ ). Only cell neighbors where the mask $M$ applies are taken into account. Each cloudy cell is associated with a local maximum by following the steepest gradient, and this process is iterated until a full list of fragments and their local maxima is generated.

The second step identifies which of the fragments can be merged. We have sketched this procedure in Fig. 1. Here, we make use of cols, which in our case are the three-dimensional equivalent of mountain passes. More specifically, a value of $\psi$ at a col is determined as follows: We consider two clouds (or two cloud fragments if merged) $C_{1}$ and $C_{2}$ that share a boundary (in principle, clouds can share multiple boundaries, corresponding to multiple cols). Subsequently, we consider the set of cells on $C_{1}$ that border $C_{2}$, denoted here as $C_{1,2}$. The value of the field $\psi$ that is highest in $C_{1,2}$ is denoted as $\psi_{1,2}$. In the same way, a value $\psi_{2,1}$ is determined on the other side of the boundary. The lowest of these values is denoted as the col value $\psi_{c}$.

The merging criterion is then based on two other values: 1) $\psi_{l}$, which is the lowest of the two local maxima corresponding to the two fragments, and 2) $\psi_{m}$, which is the lowest value anywhere on the two fragments. The criterion used for merging is

$$
\frac{\psi_{l}-\psi_{c}}{\psi_{l}-\psi_{m}} \leq f .
$$

Here, $f$ is a tunable cutoff parameter between 0 and 1 . Equation (1) implies that we use the relative importance of a local maximum $\psi_{l}$ to determine which clouds qualify as separate entities (Fig. 1). This is a similar problem to deciding which mountain peaks to give a separate label on a map. The cutoff parameter is set to 0.7 in our case. Visual inspection showed this value was suitable for tracking a number of individual cores (not shown). The method of Carr et al. (2003) is more general and allows for interactive exploration of a suitable merging criterion. For the merging procedure, we start with the highest col and process the cols in descending order.

\section{Results}

\section{a. Sub-cloud-layer circulation and cloud-layer fluxes}

With increasing $u_{*}$, the averaged zonal velocity $\overline{\langle u\rangle}$ decreases in magnitude, indicating weakened subcloud layer easterly wind, and the zonal wind shear across the top of subcloud layer $(\sim 0.6 \mathrm{~km})$ increases (Fig. 2a). Here, the angle brackets and the overbar indicate 

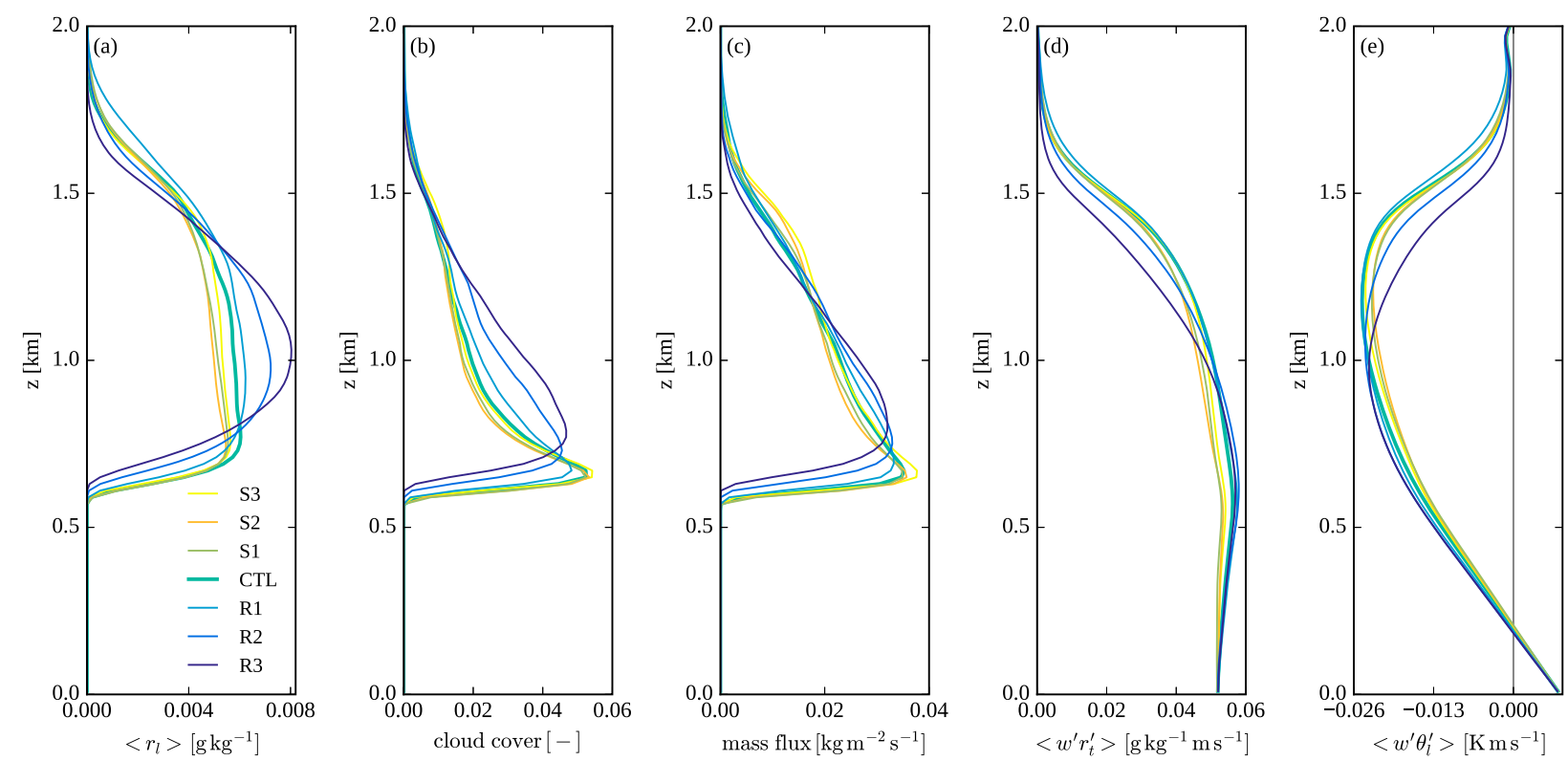

FIG. 3. Profiles of horizontally and temporally (last $2 \mathrm{~h}$ ) averaged (a) liquid water mixing ratio, (b) cloud cover, (c) mass flux in the cloudy region $\left(r_{l} \geq 0.01 \mathrm{~g} \mathrm{~kg}^{-1}\right)$, (d) vertical turbulent flux of total water mixing ratio, and (e) vertical turbulent flux of liquid water potential temperature.

horizontal and temporal (last $2 \mathrm{~h}$ ) averages, respectively. Intensifying surface friction, with increasing $u_{*}$, decreases (increases) the amount of moisture in the subcloud (cloud) layer because of stronger vertical moisture transport (Fig. 2b). Similarly, intensified surface friction increases (decreases) the liquid water potential temperature in the subcloud (cloud) layer (Fig. 2c), thus reducing the subcloud-layer relative humidity (not shown). These two series of profiles indicate that intensified surface friction transports more moisture upward to the cloud layer and more heat downward into the subcloud layer, because of the increased subcloudlayer entrainment (otherwise called boundary layer entrainment; Kim et al. 2006; Pino et al. 2003, 2006).

The impact of intensified surface friction is stronger in the vertical distribution of liquid water mixing ratio (Fig. 3a). While the control and weaker-surface-friction (S1-S3) cases display a two-layer structure, conditionally unstable cloud and stable trade inversion layers, stronger-surface-friction cases (R1-R3) show an asymmetric parabolic distribution of liquid water. Intensifying surface friction decreases $r_{l}$ below $z=0.8 \mathrm{~km}$ and above $z=1.4 \mathrm{~km}$ and increases $r_{l}$ between the two levels. Specifically, the R2 and R3 cases show a highly parabolic distribution peaking slightly above $1 \mathrm{~km}$. This latter change cannot be explained only by the increase of subcloud-layer height $z_{i}$ (where the minimum of virtual potential temperature flux occurs), which is only of a few tens of meters (Table 1), because of the increased subcloud-layer entrainment with intensified friction (Kim et al. 2006; Pino et al. 2003, 2006; see below).

Cloud cover (defined as the fraction of cloudy grid points at each level) shows a similar trend: increasing $u_{*}$ generates more parabolic and higher cloudiness at higher levels $(z>0.7 \mathrm{~km}$; Fig. 3b). With intensifying surface friction, the mass flux in cloudy regions $\left(r_{l} \geq\right.$ $0.01 \mathrm{~g} \mathrm{~kg}^{-1}$ ) increases at $z=0.75-1.2 \mathrm{~km}$ and decreases above $z=1.2 \mathrm{~km}$ compared to the control case (Fig. 3c). On the other hand, the weak-surface-friction cases (S2 and S3) show increased mass flux at $z=1.2-1.6 \mathrm{~km}$, implying deeper clouds above convective cells. With increasing $u_{*}$, the turbulent fluxes of heat and moisture increase in the subcloud layer and in the lower cloud layer below $z=0.9 \mathrm{~km}$ (Figs. 3d,e). Above that level, both turbulent fluxes in the R2 and R3 cases quickly decrease with increasing height, when compared to the control case, possibly because of the deformation of clouds there (see below for a more detailed discussion of the role of shear and cloud tilting).

Varying $u_{*}$ also affects momentum transport, and the affected momentum flux and variance are presented in appendix A for reference. The momentum flux is substantially altered by changing surface friction as expected, but it does not completely obey an eddydiffusion mass flux perspective, as recently emphasized by Schlemmer et al. (2017).

Figure 4 shows the fields of vertical velocity in the subcloud and cloud layers (at $z=250$ and $750 \mathrm{~m}$, 

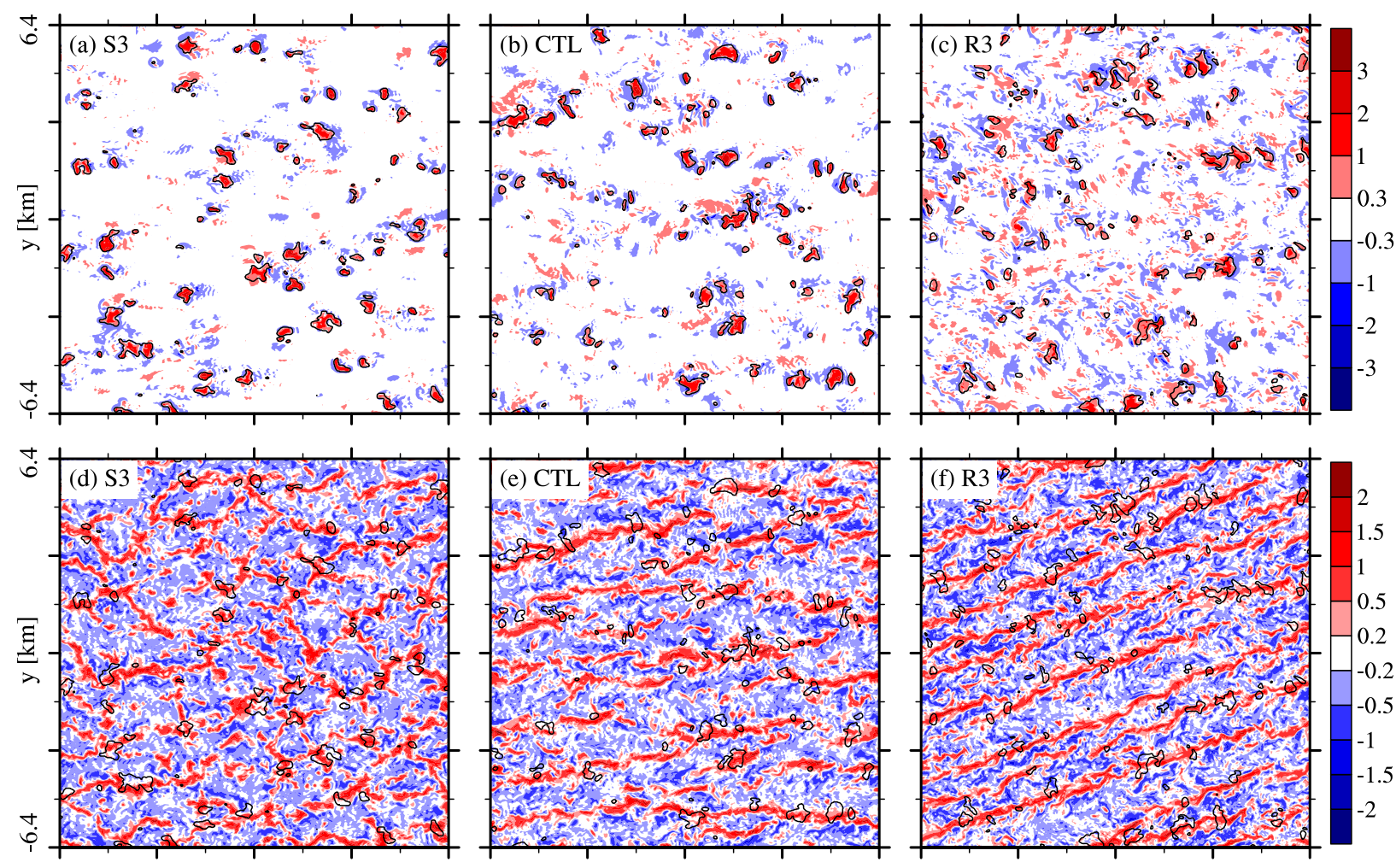

$-6.4$

$\mathrm{x}[\mathrm{km}]$

$6.4-6.4$

$\mathrm{X}[\mathrm{km}]$

$6.4-6.4$

$\mathrm{x}[\mathrm{km}]$

6.4

FIG. 4. Fields of vertical velocity at $6 \mathrm{~h}$ and $z=750 \mathrm{~m}$ in the (a) S3, (b) CTL, and (c) R3 cases. (d)-(f) As in (a)-(c), but at $z=250 \mathrm{~m}$. Black contours of $0.01 \mathrm{~g} \mathrm{~kg}^{-1}$ liquid water mixing ratio at $z=750 \mathrm{~m}$ are added in all the panels.

respectively) overlapped by $0.01 \mathrm{~g} \mathrm{~kg}^{-1}$ contours of $r_{l}$ at $z=750 \mathrm{~m}$ in the S3, CTL, and R3 cases. While convective cells are dominant in the smooth $\mathrm{S} 3$ case, convective rolls dominate the subcloud layer in the control to R3 cases, indicating that shear is already important in the CTL case. In the $\mathrm{S} 2$ case, convective cells that are slightly stretched in the $x$ direction appear, and intermediate structures between cells and rolls appear in the S1 case (not shown). The transition between the cell and roll modes seems to occur in the range of $-z_{i} / L=15-20$, as recently shown by Salesky et al. (2016). Here, the Obukhov length $L$ is defined by $-u_{*}^{3} T_{v} / \kappa g Q_{v 0}$, where $T_{v}$ is a reference mean virtual temperature at the lowest grid level, $\kappa$ is the von Kármán constant (0.4), $g$ is the gravitational acceleration $\left(9.8 \mathrm{~m} \mathrm{~s}^{-2}\right)$, and $Q_{v 0}$ is a kinematic virtual temperature flux at the surface (Foken 2006). While clouds in the S3 case appear above the intersections of cellular branches or branches themselves (Fig. 4d), most clouds in the CTL and R3 cases appear above the rising branches of convective rolls (Figs. $4 \mathrm{e}, \mathrm{f})$. We note that intensified surface friction and strengthened convective rolls generate more clouds above the rising branches of convective rolls. The abundance of unsaturated updraft and downdraft structures (especially in the $\mathrm{R} 3$ case) is also notable. These structures are generated by gravity waves or are left below rising active clouds or dissipating updrafts $(w>$ $0 \mathrm{~m} \mathrm{~s}^{-1}$ ), originated from forced clouds (confirmed in an animation of $w$ and $r_{l}$ ). Thus, the increased number of unsaturated updraft structures and forced clouds in the R3 case implies again that intensified surface friction generates more clouds in the lower cloud layer. We note that this cloud number change may be due not only to changes in the organization of convection but also to slight changes in the mean subcloud-layer state. However, the increased wind shear across $z_{i}$ and subcloudlayer entrainment generates a drier and warmer subcloud layer (Fig. 2) and thus lower subcloud-layer relative humidity (not shown), which should reduce the small forced or passive cloud cover. In fact, we observe the opposite behavior, with increased cloud number and cloud cover at higher wind shear. We thus conclude that the higher small cloud frequency in the intensified surface friction is purely a dynamical effect.

The $x-z$ cross-sectional views of clouds and zonal velocity show more drastic differences between the smooth, control, and rough cases (Fig. 5). In all cases, clouds are connected to subcloud-layer thermals, indicated by red contours of $0.8 \mathrm{~m} \mathrm{~s}^{-1}$ vertical velocity in 


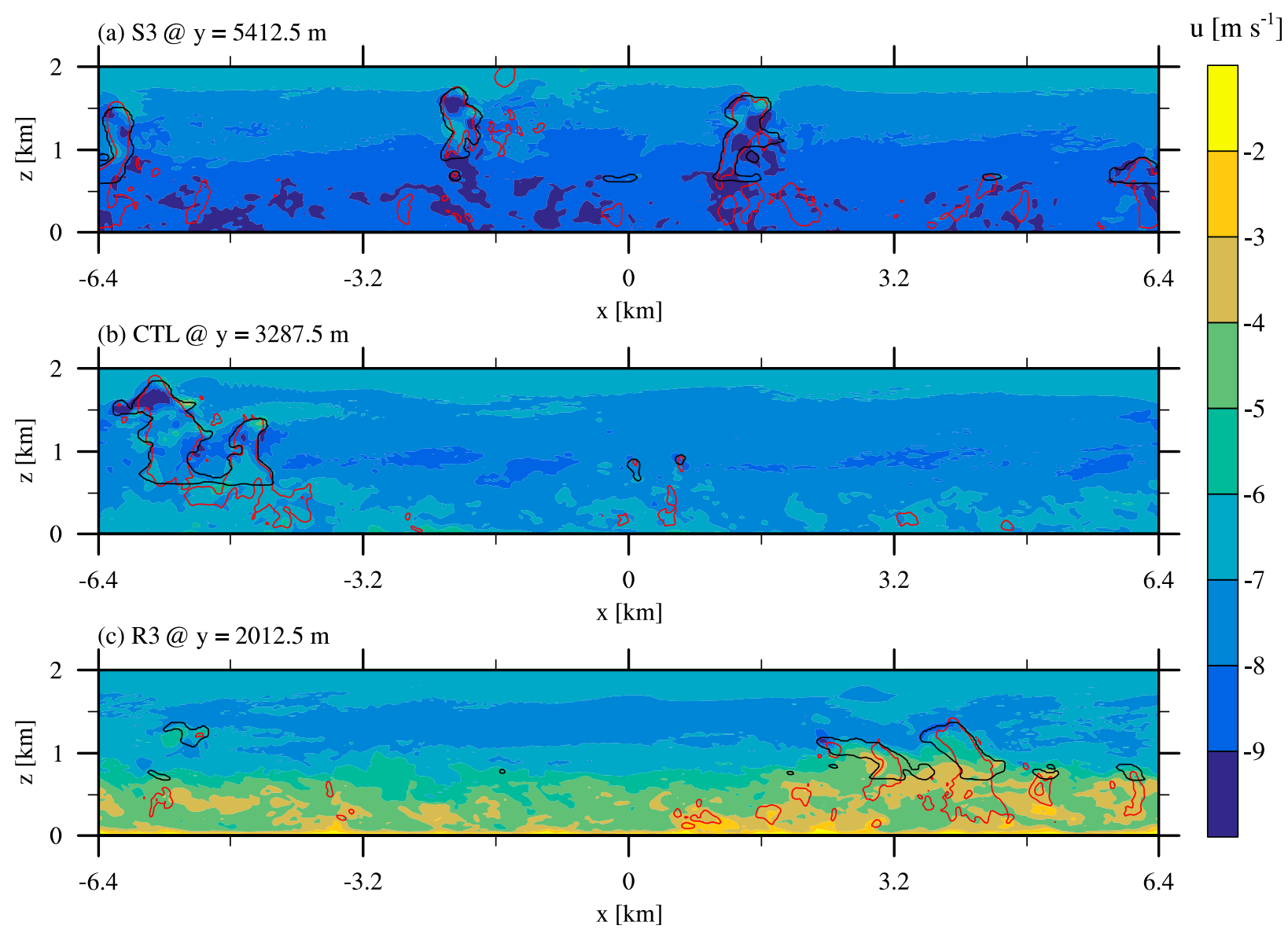

FIG. 5. Fields of zonal velocity at $6 \mathrm{~h}$ in the $x-z$ plane in the (a) S3, (b) CTL, and (c) R3 cases. Black contours of $0.01 \mathrm{~g} \mathrm{~kg}^{-1}$ liquid water mixing ratio and red contours of $0.8 \mathrm{~m} \mathrm{~s}^{-1}$ vertical velocity are added.

Fig. 5. Groups of thermals appear in the cellular branches of convective cells (Fig. 5a) or uprising branches of convective rolls (Figs. 5b,c). Many thermals in the roll-dominated cases are located in low-zonalvelocity regions (e.g., $u>-7 \mathrm{~ms}^{-1}$ in the CTL and $u>-4 \mathrm{~m} \mathrm{~s}^{-1}$ in the $\mathrm{R} 3$ cases), but the thermals' location and zonal velocity is much less correlated in the celldominated S3 case. Clouds in the S3 case are typically upright or slightly tilted in the upstream direction (here, to the east; Fig. 2a). Similarly, clouds in the CTL case tend to be upright. In the R3 case, however, clouds are strongly tilted in the downstream direction (here, to the west), and the vertical extent of clouds is strongly suppressed compared to the smoother cases. We note that the updraft-merged structures (e.g., red contours at $x=$ $4 \mathrm{~km}$ and $z=1 \mathrm{~km}$ in Fig. $5 \mathrm{c}$ ) are also tilted downstream, but they are less tilted than clouds (black contours). The difference of the tilting between the updrafts and scalars (e.g., moisture) may be a challenge for the parameterization of convective momentum transport and cloud cover.
The slanted clouds are suspected to induce different mass, heat, and moisture flux profiles (Fig. 3; see section 3b). To further evaluate this, we turn to conditional flux sampling. The profiles of conditionally sampled variables over updraft (plus-sign subscript where $w>0 \mathrm{~m} \mathrm{~s}^{-1}$ ) and downdraft (minus-sign subscript where $w<0 \mathrm{~m} \mathrm{~s}^{-1}$ ) regions, respectively, illustrate the systematic changes with intensifying surface friction (Fig. 6). Intensified surface friction increases the fraction of updrafts in the subcloud and lower cloud layers (below $z=1 \mathrm{~km}$ ). This change in the dynamics leads to an increase in cloud fraction. In the S1-S3 and CTL cases, the fraction of updrafts decreases with height below $z=0.4 \mathrm{~km}$ (less than 0.4 in the subcloud inversion layer) because of the dissipation of thermals in the stable and weakly subsiding environment. The updraft fraction in the R2 and R3 cases, however, increases with height above $z=0.25 \mathrm{~km}$, and this increase is attributable to the strengthened roll circulation and higher probability of surface-layer thermals rising through the uprising branches. The vertical velocity of the conditionally averaged updrafts decreases while the counterpart 

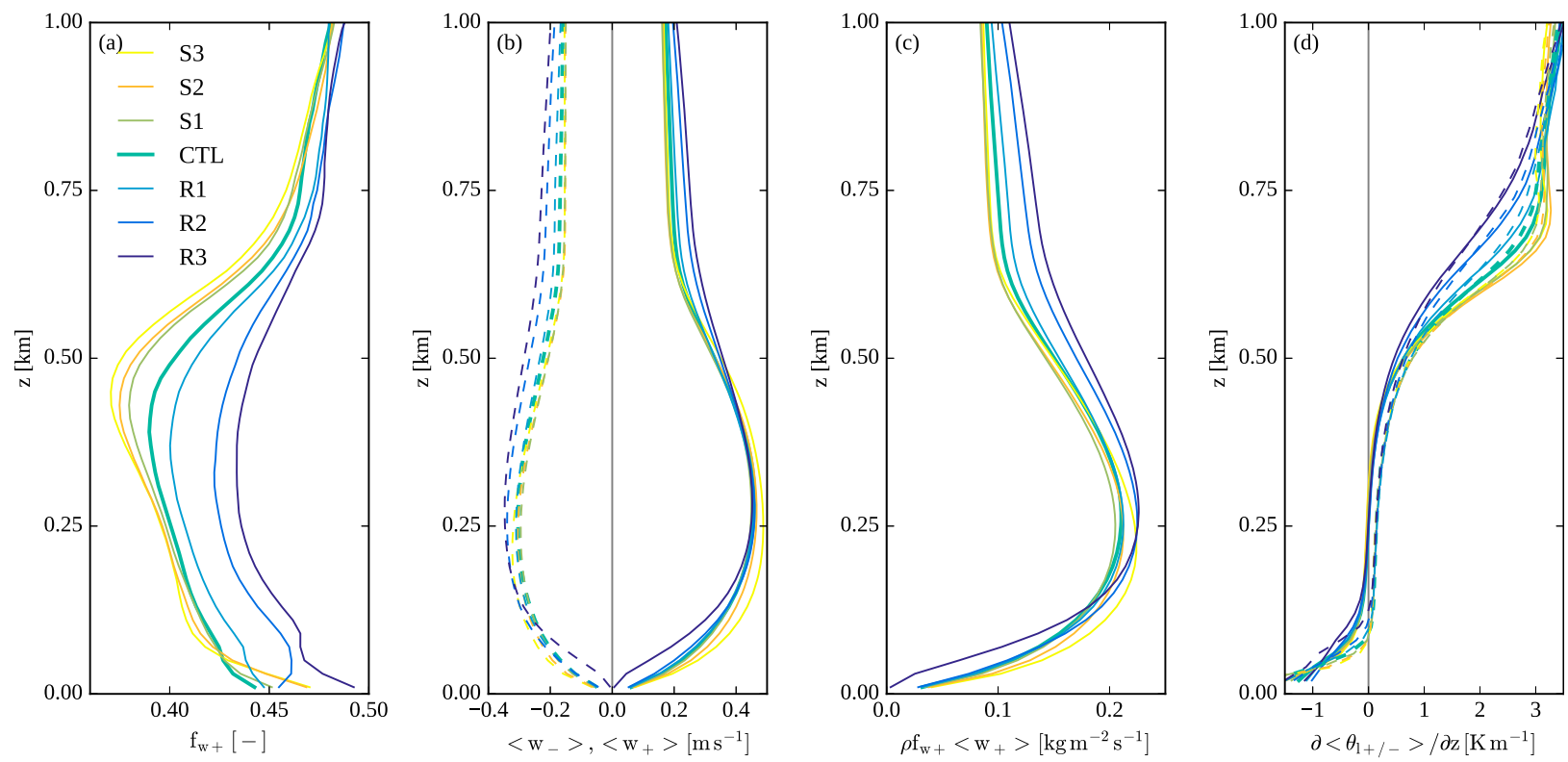

FIG. 6. Profiles of horizontally and temporally (last $2 \mathrm{~h}$ ) averaged (a) fraction of updraft region, (b) vertical velocity conditionally averaged over updraft (solid) and downdraft (dashed) regions, (c) mass flux in the updraft region, and (d) the vertical gradient of liquid water potential temperature, averaged over updraft (solid) and downdraft (dashed) regions.

(downdraft vertical velocity) increases with intensifying surface friction (Fig. 6b). Despite the weaker updraft intensity in the R2 and R3 cases, the updraft mass flux increases with intensifying surface friction because of the increased updraft fraction, and the flux increase is distinct in the upper subcloud and lower cloud layers (Fig. 6c).

We suspect that more surface-layer thermals in the roll-dominated cases (R2 and R3) rise through the uprising branches of convective rolls because of the increased updraft fraction, experiencing a locally less stable thermal environment (solid lines in Fig. 6d). Instead, in the weaker-circulation (S1, CTL, and R1) or cell-dominated (S2 and S3) cases, more surface-layer thermals are exposed to more stable and weakly subsiding environment (dashed lines in Fig. 6d) because of the increased fraction of downdrafts, then being dissipated before reaching the cloud layer. Another possibility is that thermals in the roll-dominated cases are less exposed to entrainment and/or detrainment because of their roll organization, protecting against dissipation. Furthermore, the warmer subcloud layer and cooler cloud layer in the R2 and R 3 cases decrease the thermal stratification more than in the other cases, helping thermals rise through the subcloud inversion layer and erode the thermal inversion again.

\section{b. Clouds' characteristics}

All the clouds at the 12 time instants, every $10 \mathrm{~min}$ in the last $2 \mathrm{~h}$, are identified using the method described in section 2b. As seen in Fig. 4, a larger number of clouds are identified in the R2 and R3 cases $\left(1.38-1.41 \mathrm{~km}^{-2}\right.$ per instant) compared to the smoother cases where $\sim 1.15$ clouds appear at each time instant and per square kilometer (Table 1). Figure 7 shows the identified clouds with their volume being shaded at the last time instant (6h) in the S3, CTL, and R3 cases. The cloud identification method identifies adjacent but different clouds above convective cells (Fig. 7a) or above convective rolls (Figs. $7 b, c)$. The increasing number of clouds with intensifying surface friction is confirmed again at the level $z=750 \mathrm{~m}$ in Fig. 7. The $x-z$ cross-sectional view of the clouds in Fig. 8 illustrates the complex shapes of clouds and subcloud-layer thermals (e.g., Heus and Jonker 2008). The method in this study separates different clouds even though they are weakly connected. For example, two deep clouds in the CTL case at $x=-5.6$ and $-4.6 \mathrm{~km}$, respectively, are actually connected, but they are identified as two different clouds (Fig. 8b). More information (e.g., volume, depth, and tilting in the $x$ direction) on each cloud are added in Fig. 8 to compare with the statistics in Fig. 9.

The histograms of clouds binned by volume, depth, and the degree of cloud tilting in the $x$ and $y$ directions are plotted in Fig. 9. The volume (depth) histograms use 10 logarithmically spaced bins bounded by their minimum, $1.25 \times 10^{-5} \mathrm{~km}^{3}(20 \mathrm{~m})$, and maximum, $0.81 \mathrm{~km}^{3}$ $(1460 \mathrm{~m})$. The histograms of cloud tilting in the $x(y)$ direction use 10 linearly spaced bins bounded by -5.23 

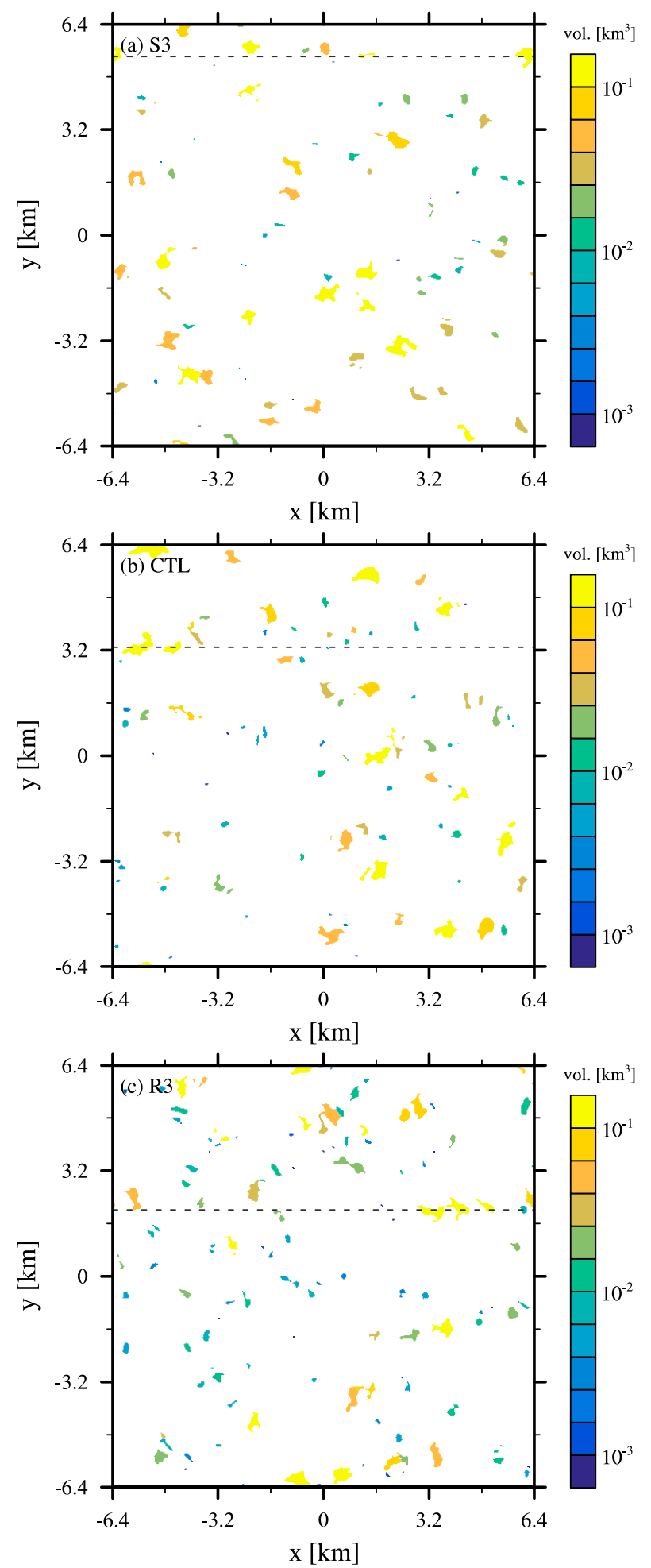

FIG. 7. Fields of identified clouds at $6 \mathrm{~h}$ and at $z=750 \mathrm{~m}$ in the (a) S3, (b) CTL, and (c) R3 cases. Color shading indicates the volume of each cloud. Gray dashed lines mark the corresponding $x-z$ planes in Fig. 8.
$(-4.44)$ and 4.20 (3.41). The volume of each cloud is calculated as the number of occupied grid points multiplied by the volume of single grid $(\Delta x \Delta y \Delta z)$. The volume histograms show peaks at the smallest bin (smaller than 3 grid boxes) and at the bin covering $3.2 \times 10^{-3}-9.6 \times 10^{-3} \mathrm{~km}^{3}$. The latter peaks correspond to developing clouds (like the easternmost yellow cloud in Fig. 8c), larger than forced or justovershooting clouds $\left(\geq 10^{-4} \mathrm{~km}^{3}\right)$ but smaller than mature deep clouds $\left(\sim 0.1 \mathrm{~km}^{3}\right)$. With intensifying surface friction, the number of clouds smaller than $0.1 \mathrm{~km}^{3}$ increases, and the number increase is distinct for the small clouds $\left(<3.2 \times 10^{-3} \mathrm{~km}^{3}\right)$ in the R2 and R3 cases. Cloud depth is simply calculated as the difference between the highest grid's top height and lowest grid's bottom height in each cloud. The depth histograms peak at the bin covering 171-262 $\mathrm{m}$ in the S2 case and at the bin covering $262-403 \mathrm{~m}$ in all the other cases. Again, intensifying surface friction generates more clouds across all bins except the largest one, and the gap between the R2 and R3 cases and the other cases is dominant. We note that cloud number in the $\mathrm{S} 3$ case is larger than that in any other case at the depth bins larger than $619 \mathrm{~m}$.

The tilting of every cloud is then quantified. To do so, the center of mass in liquid water mixing ratio is calculated at all levels reached by each cloud, and the vertical gradient of the center of mass in the $x$ and $y$ directions is calculated using the least squares polynomial fit. The histograms of cloud tilting in the $x$ direction show peaks at $\sim-1$ in the $\mathrm{R} 2$ and $\mathrm{R} 3$ cases and $\sim 0$ in the other cases; this confirms the slanted (upright) clouds in the R3 (S3 and CTL) cases in Figs. 5 and 8. The histograms also capture the fact that the S2 and S3 cases have more clouds weakly tilted in the upstream direction at tilting bins 1 and 2 (Fig. 5a). Figure 9d illustrates that more clouds are slightly tilted in the positive $y$ direction (here, to the north). This is because the Coriolis force and friction turn mean flow anticlockwise downward into the subcloud layer (Ekman spiral; Fig. A1a) and the northward tilting increases with intensifying surface friction but less distinct than in the $x$ direction.

To investigate the vertical distribution of clouds and mass flux, horizontal area $\left(N_{c} \Delta x \Delta y\right.$, where $N_{c}$ is the number of grid points occupied by each cloud) and vertical velocity averaged over the horizontal area of the individual clouds are calculated at all levels and the histograms logarithmically binned by horizontal area at all levels are plotted in Fig. 10. The horizontal cloud area bins are bounded by its minimum of $625 \mathrm{~m}^{2}$ and maximum of $1.54 \times 10^{6} \mathrm{~m}^{2}(0.94 \%$ of the horizontal domain area). The second row in Fig. 10 shows the histograms weighted by horizontal area (i.e., where every bin count 

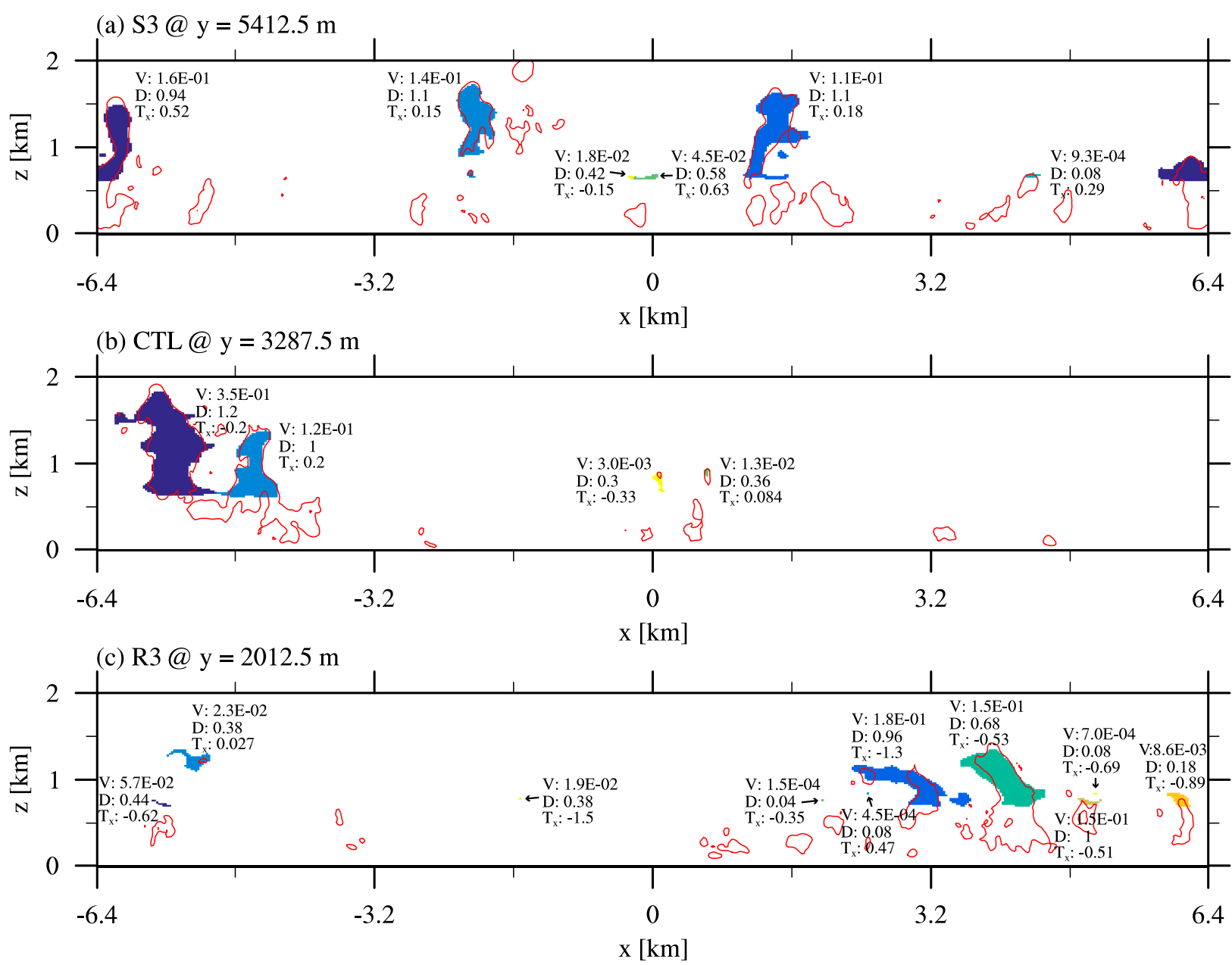

FIG. 8. Fields of identified clouds at $6 \mathrm{~h}$ in the $x-z$ plane in the (a) S3, (b) CTL, and (c) R3 cases. All the clouds are randomly renumbered for better visualization. Red contours of $0.8 \mathrm{~m} \mathrm{~s}^{-1}$ vertical velocity are added. Volume, depth, and the degree of tilting in the $x$ direction of each cloud are added to compare with the statistics in Fig. 9.

is multiplied by its horizontal area to represent cloud cover). In calculating the histograms in the third row of Fig. 10, every bin count is multiplied by its air density, horizontal area, and vertical velocity to represent the contribution of each size bin to cloud mass flux.

In the $\mathrm{S} 1-\mathrm{S} 3$ cases, more clouds rise to higher levels with decreasing surface friction (Figs. 10a-c). In the R1-R3 cases, cloud base rises, as the subcloud-layer height increases with surface friction and becomes drier with increased subcloud-layer entrainment, but fewer clouds rise to the upper cloud layer (e.g., $z=1.2-1.5 \mathrm{~km}$; Figs. 10e-g). The maximum peak of cloud number shifts from 0.1 (S3) to $0.05 \mathrm{~km}^{2}$ (R3), indicating that intensifying surface friction generates more small clouds in the lower cloud layer (e.g., below $z=0.9 \mathrm{~km}$; Figs. 4 and 7). However, the larger clouds contribute more to the total cloud cover and cloud mass flux compared to the small clouds.
Clouds larger than $0.1 \mathrm{~km}^{2}$ mostly contribute to cloud cover slightly above cloud base $(\sim 0.6 \mathrm{~km})$ in the $\mathrm{S} 1-\mathrm{S} 3$ and CTL cases (Figs. 10h-k). Instead, clouds well above the cloud base (e.g., $z \geq 0.65 \mathrm{~km}$ ) contribute more to cloud cover in the R2 and R3 cases with more smaller clouds $\left(<0.1 \mathrm{~km}^{2}\right)$ being left below (Figs. 10m,n). This kind of trend matches the highly parabolic $r_{l}$ distribution in the R2 and R3 cases (Fig. 3a). The histograms representing cloud mass flux show a similar trend with the cloud-cover histograms except more distinct contribution of clouds at higher level. Again, with intensifying surface friction, the center of the histograms shifts to higher levels, indicating that cloud tilting induces more cloud mass flux at higher level (e.g., above $z=0.8 \mathrm{~km}$; Figs. $10 \mathrm{o}-\mathrm{u}$ ).

The entrainment and detrainment rates across clouds $\left(r_{l}=0.01 \mathrm{~g} \mathrm{~kg}^{-1}\right)$, following the method of Romps (2010; i.e., the so-called direct entrainment rates), confirm the 

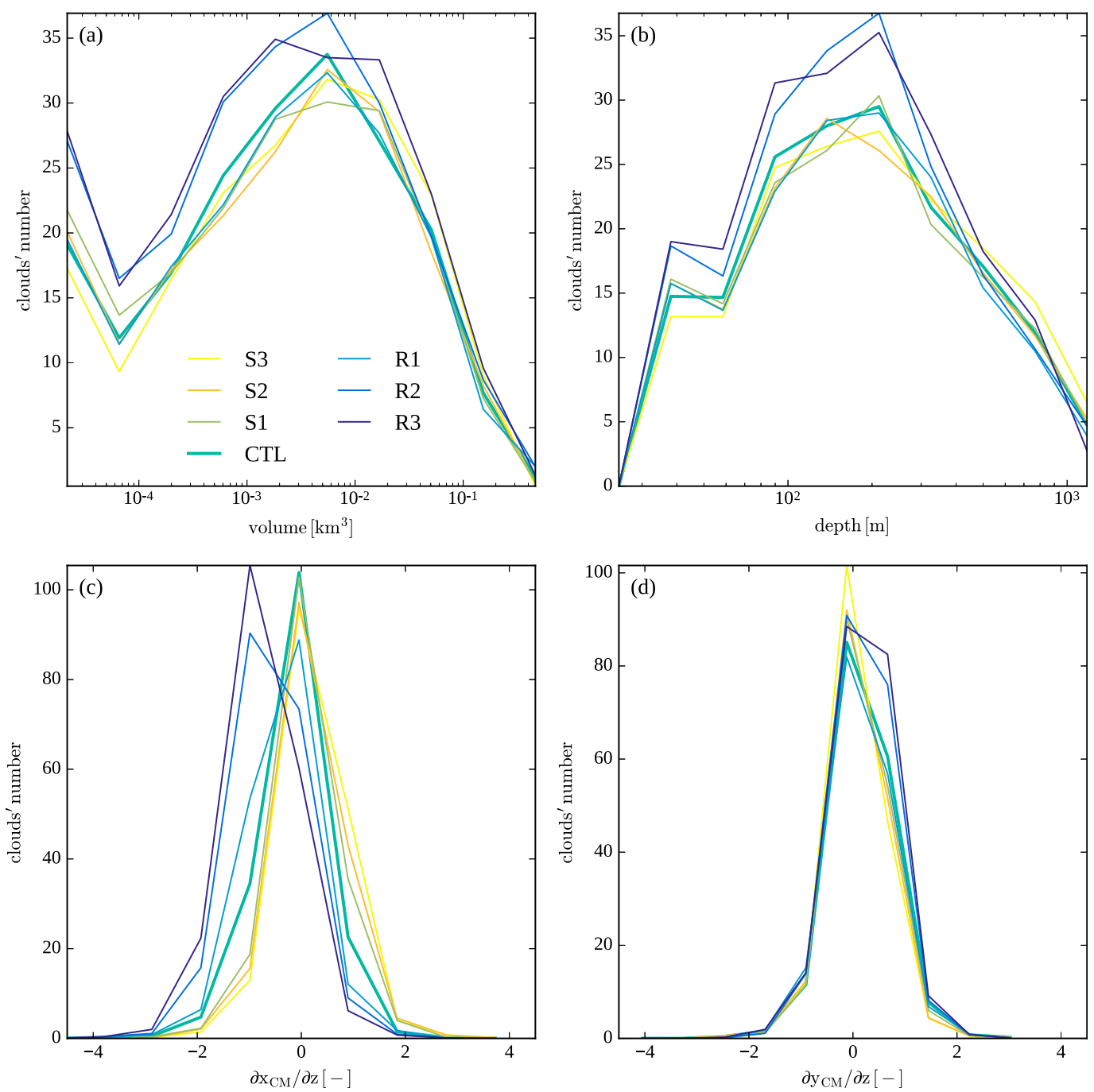

FIG. 9. Histograms of identified clouds binned by their (a) volume, (b) depth, (c) tilting in the $x$ direction, and (d) tilting in the $y$ direction. Tilting is quantified by the vertical gradient of center of mass (liquid water mixing ratio).

difference in cloud fraction and cloud mass flux with increasing $u_{*}$ (Fig. 11). In the S3 and CTL cases, entrainment minus detrainment rates, corresponding to net entrainment into clouds (proportional to $d \log \left(M_{c}\right) / d z$, where $M_{c}$ is cloud mass flux), has a positive peak at $z_{i}$, a negative peak at $z \sim 0.7 \mathrm{~km}$, and a smaller negative peak in the trade inversion layer (Fig. 11a). While the positive and negative peaks at $\sim 100 \mathrm{~m}$ above $z_{i}$ are attributable to the generation and dissipation of small clouds, respectively, the peak in the trade inversion layer is related to deeper clouds, actively detraining at their tops. In the R3 case, however, the positive peak is smaller than in the other two cases, and only one negative peak appears at $z \sim 1.1 \mathrm{~km}$ (well above $z_{i}$ ). The fractional detrainment rate in the R3 case at $z \sim 0.75 \mathrm{~km}$ is smaller than that in the other cases (Fig. 11c), indicating that fewer small clouds terminate at this height. Instead, both the fractional entrainment and detrainment rates in the R3 case at $z \sim 1.2 \mathrm{~km}$ are larger than those in the other cases, implying more active airflow across the surfaces of tilted clouds (Figs. 11b,c). Thus, the detrainment of clouds in the R3 case depends more on (local) shear instability but less on environmental stability.

\section{Summary and conclusions}

The role of surface friction on shallow convection has been investigated using a series of large-eddy simulations with varying surface friction velocity and using a cloud identification algorithm. In reality, such strong surface friction can be due to increased ocean waves or canopy drag (Sullivan et al. 2014; Patton et al. 2015). 


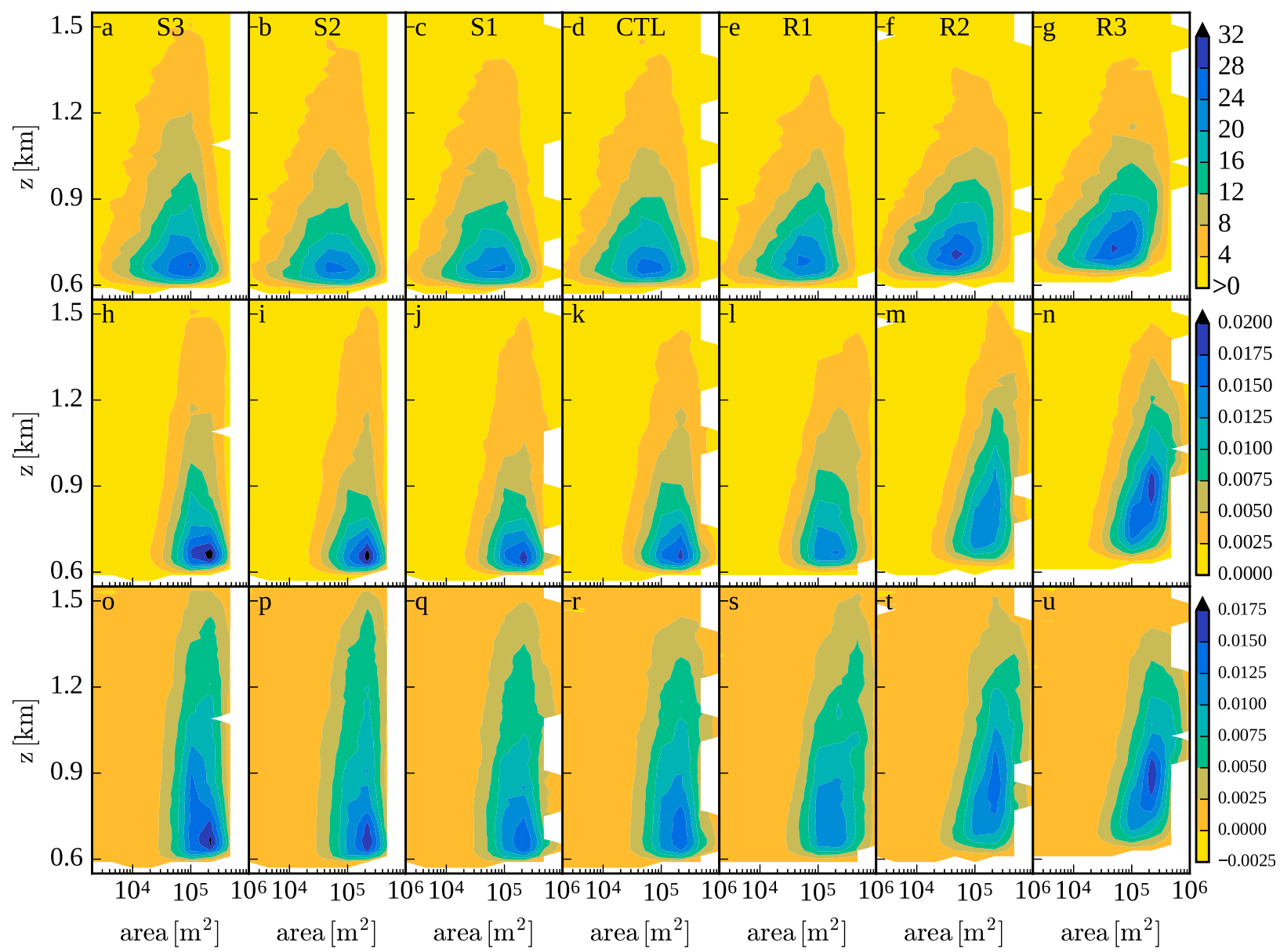

FIG. 10. Histograms of clouds binned by their horizontal area, calculated at all levels, in the (a) S3, (b) S2, (c) S1, (d) CTL, (e) R1, (f) R2, and (g) R3 cases. (h)-(n) Histograms weighted by horizontal area to illustrate binned cloud cover. (o)-(u) Every bin count of the histograms is multiplied by its air density, horizontal area, and vertical velocity to illustrate binned cloud mass flux.

With intensifying surface friction, convective rolls dominate over cells, and secondary overturning circulation, represented by updraft mass flux here, becomes stronger in the subcloud layer and is accompanied by more frequent small clouds despite the lower relative humidity in the subcloud layer. Intensified surface friction transports more moisture upward and more heat downward between the subcloud and lower cloud layers. Highly intensified surface friction in the two most intense cases induces more liquid water and larger cloud mass flux in the lower cloud layer (e.g., $0.8<z<$ $1.2 \mathrm{~km}$ ), while the classical two-layer structure is kept in the weaker-surface-friction cases. Identifying and analyzing individual clouds reveals that intensified surface friction increases the number of clouds and the degree of tilting in the downstream direction. Cloud tilting induces more cloud mass flux at higher level (e.g., $0.8<z<$ $1.2 \mathrm{~km}$ ), and this trend corresponds to the profiles of directly measured entrainment and detrainment rates, showing less detrainment in the lower cloud layer. Opposite to the lower cloud-layer behavior, cloud tilting induces less cloud mass flux and less moisture and heat transport at higher levels (e.g., $z>1.2 \mathrm{~km}$ ), that is, in the cloud inversion layer. This trend is explained by the increased entrainment rate with cloud tilting with higher wind shear. In other words, tilted sheared clouds experience more lateral entrainment per unit height as they are tilted compared to the vertical.

We have shown that clouds are intimately connected to the subcloud-layer coherent structures: stronger surface friction leads to convective rolls (Mason and Sykes 1982; Sykes and Henn 1989), and larger wind shear across the convection layer (Kim et al. 2003), and thus tilting clouds and changing vertical fluxes of mass, heat, and moisture. A challenge is to understand the difference between the progression of cloudy updrafts and advection of liquid water. The newly condensed parts, usually tops of cloudy updrafts, accelerate air around, 

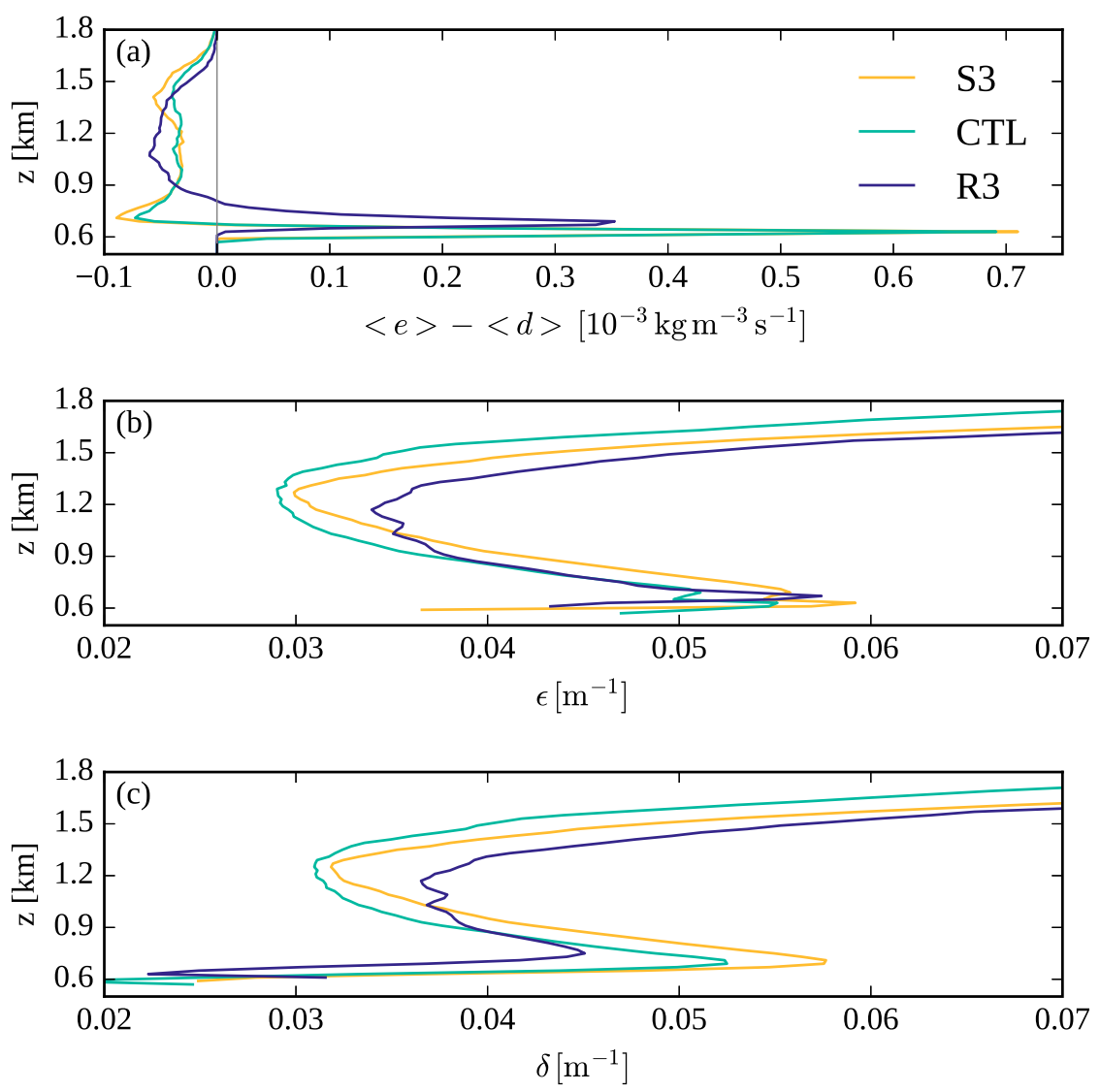

FIG. 11. Vertical profiles of directly measured (a) entrainment minus detrainment, (b) fractional entrainment rate, and (c) fractional detrainment rate (Romps 2010). We note that the entrainment and detrainment rates are dependent on the LES resolution so that they are larger than in Romps (2010), where 50-m grid resolution is used.

and the accelerated rising air pulls moisture higher up, continuing condensation. Cloudy air, detrained from or left below the successive updrafts, is passively advected and thus tilted differently. We suggest that a new shallow convection parameterization should handle the updrafts' progression and passive water advection separately. Then, more accurate parameterization of cloud cover and deepening of convection especially in response to shear will be possible.

Understanding convective momentum transport (CMT) through the convective rolls and tilted clouds is also challenging. Although accurate CMT parameterization can reduce the biases in surface winds and in tropical convection (Richter and Rasch 2008), CMT is not sufficiently understood nor accurately parameterized because CMT and convective scalar transport are quite different even in shallow convection (appendix A). Further study focusing on the impacts of wind shear on CMT and the pressure effect (Schlemmer et al. 2017) is required. In addition, more realistic simulations could be performed using a closed surface energy slab-ocean model (Tan et al.
2016), resolving atmospheric and ocean eddies together (Esau 2014) but will render the analysis more difficult, in particular the decoupling between the dynamic and thermodynamic effects. These closer-to-real-world simulations would help us better understand and parameterize the atmospheric response to surface friction change (e.g., impacts on momentum and/or scalar transport, cloud cover, energy balance, and general circulation).

Acknowledgments. The authors would like to acknowledge three anonymous reviewers for their valuable comments. This work was supported by Pierre Gentine's grants: NASA new investigator Grant NNX14AI36G, DOE Early Career Grant DE-SC0014203, and NSF CAREER and GoAmazon DE-SC0011094. We would like to acknowledge high-performance computing support from Yellowstone (ark:/85065/d7wd3xhc) provided by NCAR's Computational and Information Systems Laboratory, sponsored by the National Science Foundation. Steven Böing is funded through the NERC/MetOffice program on parameterization of con- 

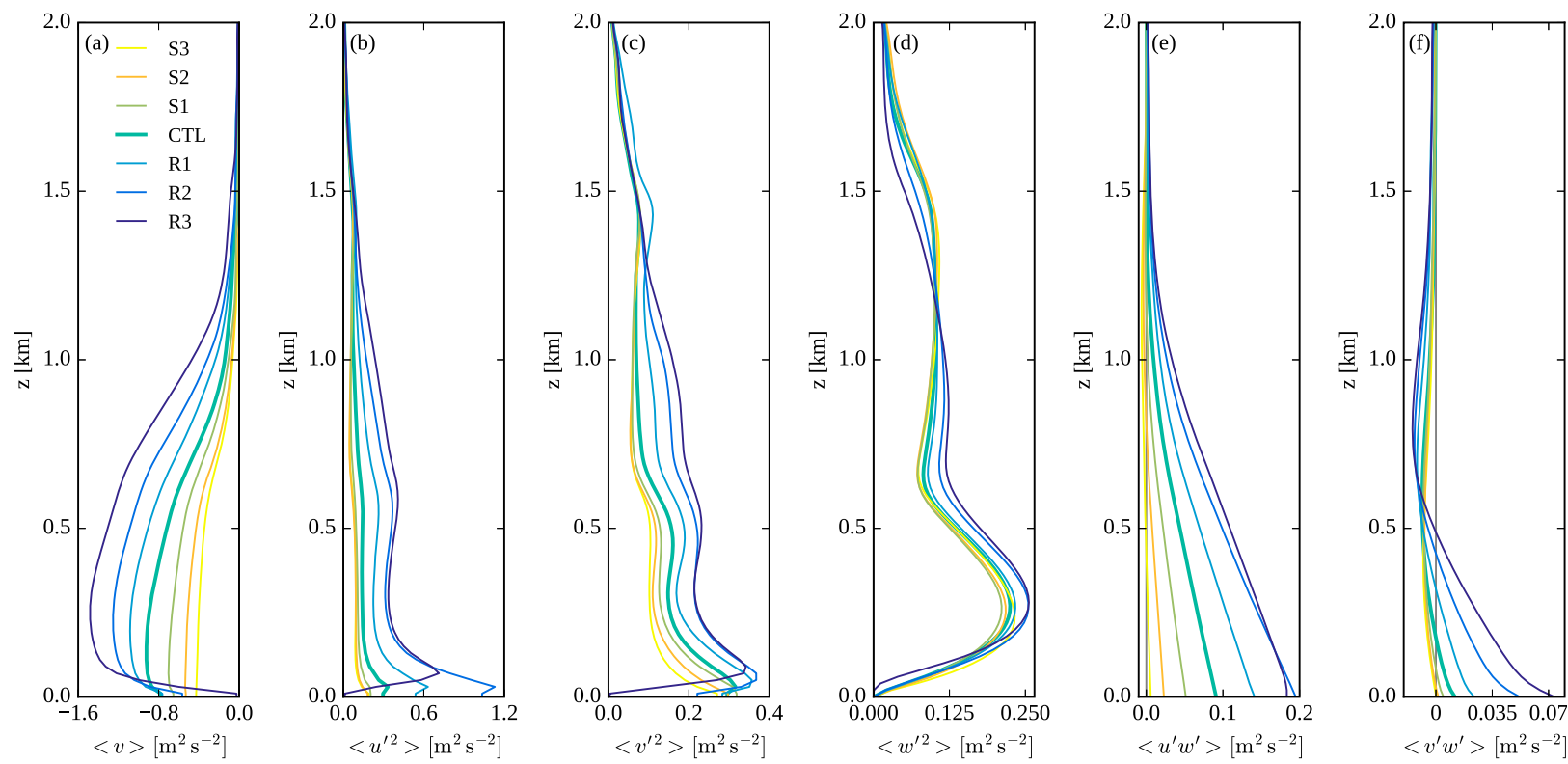

FIG. A1. Profiles of horizontally and temporally (last $2 \mathrm{~h}$ ) averaged (a) meridional velocity, (b) zonal velocity variance, (c) meridional velocity variance, (d) vertical velocity variance, and vertical turbulent fluxes of (e) zonal momentum and (f) meridional momentum.

vection across scales (ParaCon). The authors thank Michael Whitall (Met Office) for suggestions that improved the cloud identification algorithm.

\section{APPENDIX A}

\section{Impacts of Varying Surface Friction Velocity on Momentum Fluxes}

The averaged meridional velocity increases in magnitude with increasing $u_{*}$ because intensified friction rotates flow more southward (Ekman spiral) and the rotated layer becomes deeper with intensifying surface friction (Fig. A1). The zonal and meridional velocity variances show peaks at the top of surface layer (Moeng and Sullivan 1994) and at the top of subcloud layer. The two variances almost monotonically increase with $u_{*}$ in the subcloud and cloud layers except that the variances in the R3 case are smaller than those in the other cases in the surface layer. This can be attributed to too weak surfacelayer flow $\left(|\overline{\langle u\rangle}|<3 \mathrm{~m} \mathrm{~s}^{-1}\right.$ at $\left.0.1 z_{i}\right)$ in the R3 case (Fig. 2a). The vertical velocity variance has maxima in the middle of the subcloud layer (Moeng and Sullivan 1994), and in the cloud layer, it increases (decreases) with $u_{*}$ below (above) $z \sim 1.2 \mathrm{~km}$. This exactly matches the trend of vertical turbulent fluxes of heat and moisture in the cloud layer (Figs. 3d,e). However, the vertical fluxes of zonal momentum and meridional momentum increase monotonically with intensifying surface friction (Figs. A1e,f). This indicates that the momentum transport mechanism is different from the scalar transport mechanism in the cloud layer as emphasized by Schlemmer et al. (2017).

\section{APPENDIX B}

\section{Impacts of the Coriolis Force and Interactive Surface Fluxes}

A large-eddy simulation with the same setup as the CTL case but without the Coriolis force (noCF) is performed to see how much the Coriolis force affects the results. When the Coriolis force vanishes, the mean easterly is slightly decelerated. The vertical turbulent fluxes of moisture and heat increase above $z \sim 1.1 \mathrm{~km}$ (and slightly decrease in the lower cloud layer) when compared to the CTL case $\left(15^{\circ} \mathrm{N}\right.$ latitude), indicating that the Coriolis parameter has some influence on the depth of convection.

Two more simulations, where all the surface fluxes are calculated by Monin-Obukhov similarity theory (MOST), are performed to show a response of scalar (heat and/or moisture) flux to intensifying surface friction. Here, the sea surface temperature is fixed to $300.4 \mathrm{~K}$, and the surface roughness lengths are $3 \times 10^{-4}$ $\mathrm{m}$ in the MOST control (MOC) and $0.03 \mathrm{~m}$ in the MOST rough (MOR) cases, respectively. The vertical profiles of the momentum fluxes in the two cases are well matched to their counterparts (Figs. B1b,c). However, the vertical profiles of moisture and heat in the MOC and MOR cases are totally different from their counterparts (Figs. B1d,e). Especially in the MOR case, the 

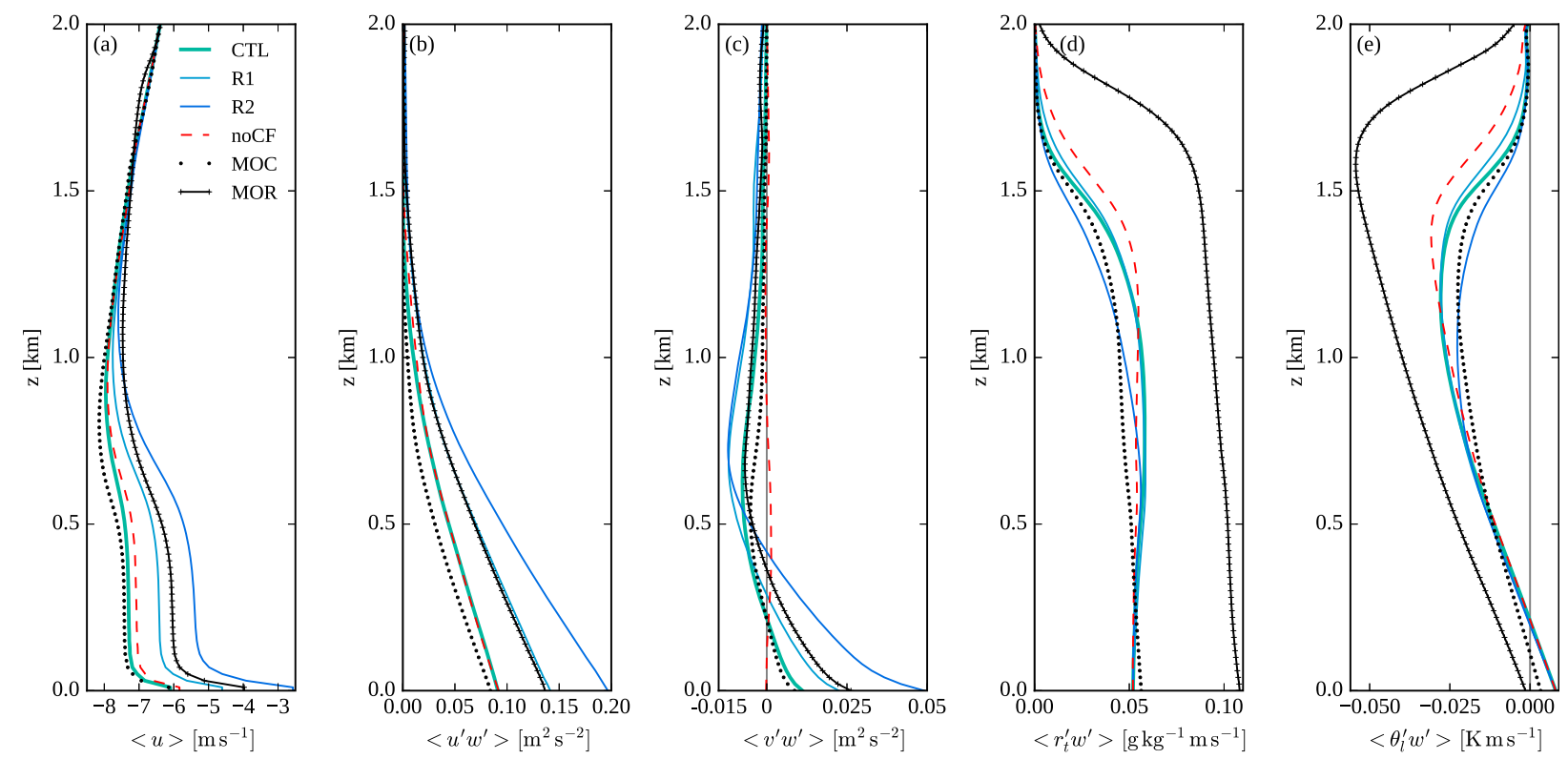

FIG. B1. Profiles of horizontally and temporally (last $2 \mathrm{~h}$ ) averaged (a) zonal velocity, and vertical turbulent fluxes of (b) zonal momentum, (c) meridional momentum, (d) total water mixing ratio, and (e) liquid water potential temperature in the CTL, R1, R2, noCF, MOC, and MOR cases.

surface flux of total water mixing ratio (moisture input) is almost twice that in the CTL case, thus inducing completely different distribution pattern of moisture. This again indicates that the setup with the fixed surface heat and moisture fluxes in this study was required to investigate the sole dynamical impact of surface friction.

\section{REFERENCES}

Bony, S., and Coauthors, 2006: How well do we understand and evaluate climate change feedback processes? J. Climate, 19, 3445-3482, https://doi.org/10.1175/JCLI3819.1.

Carr, H., J. Snoeyink, and U. Axen, 2003: Computing contour trees in all dimensions. Comput. Geom., 24, 75-94, https://doi.org/ 10.1016/S0925-7721(02)00093-7.

Couvreux, F., F. Hourdin, and C. Rio, 2010: Resolved versus parametrized boundary-layer plumes. Part I: A parametrizationoriented conditional sampling in large-eddy simulations. Bound.-Layer Meteor., 134, 441-458, https://doi.org/10.1007/ s10546-009-9456-5.

Dawe, J. T., and P. H. Austin, 2012: Statistical analysis of an LES shallow cumulus cloud ensemble using a cloud tracking algorithm. Atmos. Chem. Phys., 12, 1101-1119, https://doi.org/ 10.5194/acp-12-1101-2012.

de Rooy, W. C., and A. P. Siebesma, 2008: A simple parameterization for detrainment in shallow cumulus. Mon. Wea. Rev., 136, 560-576, https://doi.org/10.1175/2007MWR2201.1.

— , and Coauthors, 2013: Entrainment and detrainment in cumulus convection: An overview. Quart. J. Roy. Meteor. Soc., 139, 1-19, https://doi.org/10.1002/qj.1959.

Dixon, M., and G. Wiener, 1993: TITAN: Thunderstorm Identification, Tracking, Analysis, and Nowcasting-A radar-based methodology. J. Atmos. Oceanic Technol., 10, 785-797, https:// doi.org/10.1175/1520-0426(1993)010<0785:TTITAA > 2.0.CO;2.
Esau, I., 2014: Indirect air-sea interactions simulated with a coupled turbulence-resolving model. Ocean Dyn., 64, 689-705, https://doi.org/10.1007/s10236-014-0712-y.

Foken, T., 2006: 50 years of the Monin-Obukhov similarity theory. Bound.-Layer Meteor., 119, 431-447, https://doi.org/10.1007/ s10546-006-9048-6.

Gentine, P., G. Bellon, and C. C. van Heerwaarden, 2015: A closer look at boundary layer inversion in large-eddy simulations and bulk models: Buoyancy-driven case. J. Atmos. Sci., 72, 728749, https://doi.org/10.1175/JAS-D-13-0377.1.

Grant, A. L. M., and A. R. Brown, 1999: A similarity hypothesis for shallow-cumulus transports. Quart. J. Roy. Meteor. Soc., 125, 1913-1936, https://doi.org/10.1002/qj.49712555802.

Heus, T., and H. J. J. Jonker, 2008: Subsiding shells around shallow cumulus clouds. J. Atmos. Sci., 65, 1003-1018, https://doi.org/ 10.1175/2007JAS2322.1.

_ , and A. Seifert, 2013: Automated tracking of shallow cumulus clouds in large domain, long duration large eddy simulations. Geosci. Model Dev., 6, 1261-1273, https://doi.org/10.5194/ gmd-6-1261-2013.

, H. J. J. Jonker, H. E. A. Van den Akker, E. J. Griffith, M. Koutek, and F. H. Post, 2009: A statistical approach to the life cycle analysis of cumulus clouds selected in a virtual reality environment. J. Geophys. Res., 114, D06208, https://doi.org/ 10.1029/2008JD010917.

Holland, J. Z., and E. M. Rasmusson, 1973: Measurement of the atmospheric mass, energy, and momentum budgets over a 500-kilometer square of tropical ocean. Mon. Wea. Rev., 101, 44-57, https:// doi.org/10.1175/1520-0493(1973)101<0044:MOTAME>2.3.CO;2.

Johnson, J. T., P. L. MacKeen, A. Witt, E. D. W. Mitchell, G. J. Stumpf, M. D. Eilts, and K. W. Thomas, 1998: The Storm Cell Identification and Tracking algorithm: An enhanced WSR-88D algorithm. Wea. Forecasting, 13, 263-276, https:// doi.org/10.1175/1520-0434(1998)013<0263:TSCIAT>2.0.CO;2.

Katul, G. G., G. Kuhn, J. Schieldge, and C.-I. Hsieh, 1997: The ejection-sweep character of scalar fluxes in the unstable 
surface layer. Bound.-Layer Meteor., 83, 1-26, https://doi.org/ 10.1023/A:1000293516830.

Kim, S.-W., S.-U. Park, and C. Moeng, 2003: Entrainment processes in the convective boundary layer with varying wind shear. Bound.-Layer Meteor., 108, 221-245, https://doi.org/ 10.1023/A:1024170229293.

$\longrightarrow,-$, D. Pino, and J. Vilà-Guerau de Arellano, 2006: Parameterization of entrainment in a sheared convective boundary layer using a first-order jump model. Bound.-Layer Meteor., 120, 455-475, https://doi.org/10.1007/s10546-006-9067-3.

Kropfli, R. A., and N. M. Kohn, 1978: Persistent horizontal rolls in the urban mixed layer as revealed by dual-Doppler radar. J. Appl. Meteor., 17, 669-676, https://doi.org/10.1175/ 1520-0450(1978)017<0669:PHRITU>2.0.CO;2.

Kuhn, A., and S. Trömel, 2015: Intercomparison study of cloud feature extraction and tracking algorithms. Geophysical Research Abstracts, Vol. 17, Abstract 14128, http://meetingorganizer. copernicus.org/EGU2015/EGU2015-14128.pdf.

Mason, P. J., and R. I. Sykes, 1982: A two-dimensional numerical study of horizontal roll vortices in an inversion capped planetary boundary layer. Quart. J. Roy. Meteor. Soc., 108, 801-823, https://doi.org/10.1002/qj.49710845805.

Matthews, S., J. M. Hacker, J. Cole, J. Hare, C. N. Long, and R. M. Reynolds, 2007: Modification of the atmospheric boundary layer by a small island: Observations from Nauru. Mon. Wea. Rev., 135, 891-905, https://doi.org/10.1175/MWR3319.1.

Moeng, C.-H., and P. P. Sullivan, 1994: A comparison of shear- and buoyancy-driven planetary boundary layer flows. J. Atmos. Sci., 51, 999-1022, https://doi.org/10.1175/1520-0469(1994)051<0999: $\mathrm{ACOSAB}>2.0 . \mathrm{CO} ; 2$.

Nuijens, L., B. Medeiros, I. Sandu, and M. Ahlgrimm, 2015: The behavior of trade-wind cloudiness in observations and models: The major cloud components and their variability. J. Adv. Model. Earth Syst., 7, 600-616, https://doi.org/10.1002/2014MS000390.

Park, S.-B., and J.-J. Baik, 2014: Large-eddy simulations of convective boundary layers over flat and urbanlike surfaces. J. Atmos. Sci., 71, 1880-1892, https://doi.org/10.1175/JAS-D-13-0191.1.

- , P. Gentine, K. Schneider, and M. Farge, 2016: Coherent structures in the boundary and cloud layers: Role of updrafts, subsiding shells, and environmental subsidence. J. Atmos. Sci., 73, 1789-1814, https://doi.org/10.1175/JAS-D-15-0240.1.

Patton, E. G., P. P. Sullivan, R. H. Shaw, J. J. Finnigan, and J. C. Weil, 2015: Atmospheric stability influences on coupled boundary layer and canopy turbulence. J. Atmos. Sci., 73, 1621-1647, https://doi.org/10.1175/JAS-D-15-0068.1.

Pino, D., J. Vilà-Guerau de Arellano, and P. G. Duynkerke, 2003: The contribution of shear to the evolution of a convective boundary layer. J. Atmos. Sci., 60, 1913-1926, https://doi.org/ 10.1175/1520-0469(2003)060<1913:TCOSTT >2.0.CO;2.

,-- , and S.-W. Kim, 2006: Representing sheared convective boundary layer by zeroth- and first-order-jump mixed-layer models: Large-eddy simulation verification. J. Appl. Meteor. Climatol., 45, 1224-1243, https://doi.org/10.1175/JAM2396.1.

Raupach, M. R., 1981: Conditional statistics of Reynolds stress in rough-wall and smooth-wall turbulent boundary layers. J. Fluid Mech., 108, 363-382, https://doi.org/10.1017/S0022112081002164.

Richter, J. H., and P. J. Rasch, 2008: Effects of convective momentum transport on the atmospheric circulation in the Community Atmosphere Model, version 3. J. Climate, 21, 1487-1499, https://doi.org/10.1175/2007JCLI1789.1.

Romps, D. M., 2010: A direct measure of entrainment. J. Atmos. Sci., 67, 1908-1927, https://doi.org/10.1175/2010JAS3371.1.
Salesky, S. T., M. Chamecki, and E. Bou-Zeid, 2016: On the nature of the transition between roll and cellular organization in the convective boundary layer. Bound.-Layer Meteor., 163, 41-68, https://doi.org/10.1007/s10546-016-0220-3.

Schlemmer, L., P. Bechtold, I. Sandu, and M. Ahlgrimm, 2017: Uncertainties related to the representation of momentum transport in shallow convection. J. Adv. Model. Earth Syst., 9, 1269-1291, https://doi.org/10.1002/2017MS000915.

Siebesma, A. P., and J. W. M. Cuijpers, 1995: Evaluation of parametric assumptions for shallow cumulus convection. J. Atmos. Sci., 52, 650-666, https://doi.org/10.1175/1520-0469(1995)052<0650: EOPAFS $>2.0 . \mathrm{CO} ; 2$.

— , and Coauthors, 2003: A large eddy simulation intercomparison study of shallow cumulus convection. J. Atmos. Sci., 60 , 1201-1219, https://doi.org/10.1175/1520-0469(2003)60<1201: ALESIS $>2.0 . \mathrm{CO} ; 2$.

Stevens, B., and A. Seifert, 2008: Understanding macrophysical outcomes of microphysical choices in simulations of shallow cumulus convection. J. Meteor. Soc. Japan, 86A, 143-162, https://doi.org/10.2151/jmsj.86A.143.

_ C.-H. Moeng, and P. P. Sullivan, 1999: Large-eddy simulations of radiatively driven convection: Sensitivities to the representation of small scales. J. Atmos. Sci., 56, 3963-3984, https://doi.org/ 10.1175/1520-0469(1999)056<3963:LESORD>2.0.CO;2.

_ observations of nocturnal marine stratocumulus. Mon. Wea. Rev., 133, 1443-1462, https://doi.org/10.1175/MWR2930.1.

Stull, R. B., 1988: An Introduction to Boundary Layer Meteorology. Kluwer Academic, 666 pp.

Sullivan, P. P., C.-H. Moeng, B. Stevens, D. H. Lenschow, and S. D. Mayor, 1998: Structure of the entrainment zone capping the convective atmospheric boundary layer. J. Atmos. Sci., 55, 3042-3064, https://doi.org/10.1175/1520-0469(1998)055<3042: SOTEZC $>2.0 . \mathrm{CO} ; 2$.

_ J. C. McWilliams, and E. G. Patton, 2014: Large-eddy simulation of marine atmospheric boundary layers above a spectrum of moving waves. J. Atmos. Sci., 71, 4001-4027, https://doi.org/10.1175/JAS-D-14-0095.1.

Sykes, R. I., and D. S. Henn, 1989: Large-eddy simulation of turbulent sheared convection. J. Atmos. Sci., 46, 1106-1118, https://doi.org/ 10.1175/1520-0469(1989)046<1106:LESOTS > 2.0.CO;2.

Tan, Z., T. Schneider, J. Teixeira, and K. G. Pressel, 2016: Largeeddy simulation of subtropical cloud-topped boundary layers: 1. A forcing framework with closed surface energy balance. J. Adv. Model. Earth Syst., 8, 1565-1585, https://doi.org/ 10.1002/2016MS000655.

Troen, I. B., and L. Mahrt, 1986: A simple model of the atmospheric boundary layer; sensitivity to surface evaporation. Bound.-Layer Meteor., 37, 129-148, https://doi.org/10.1007/ BF00122760.

Weckwerth, T. M., T. Horst, and J. Wilson, 1999: An observational study of the evolution of horizontal convective rolls. Mon. Wea. Rev., 127, 2160-2179, https://doi.org/10.1175/ 1520-0493(1999)127<2160:AOSOTE > 2.0.CO;2.

Wicker, L. J., and W. C. Skamarock, 2002: Time-splitting methods for elastic models using forward time schemes. Mon. Wea. Rev., 130, 2088-2097, https://doi.org/10.1175/1520-0493(2002) $130<2088$ :TSMFEM $>2.0$. CO;2.

Yu, M., and C. Yang, 2017: A 3D multi-threshold, region-growing algorithm for identifying dust storm features from model simulations. Int. J. Geogr. Inf. Sci., 31, 939-961, https://doi.org/ 10.1080/13658816.2016.1250900. 